\title{
On the Performance of the Box Particle Filter for Extended Object Tracking Using Laser Data
}

\author{
Nikolay Petrov $^{1}$, Martin Ulmke ${ }^{2}$, Lyudmila Mihaylova ${ }^{1}$, Amadou Gning ${ }^{\natural}$, \\ Marek Schikora ${ }^{2}$, Monika Wieneke ${ }^{2}$ and Wolfgang Koch $^{2}$ \\ ${ }^{1}$ School of Computing and Communications, Lancaster University, United Kingdom \\ ${ }^{2}$ Department of Sensor Data and Information Fusion, Fraunhofer FKIE, Wachtberg 53343 Germany \\ ${ }^{\natural}$ Department of Computer Science, University College London, United Kingdom
}

E-mails: n.petrov@lancs.ac.uk,martin.ulmke@fkie.fraunhofer.de, mila.mihaylova@lancs.ac.uk, a.gning@cs.ucl.ac.uk

\begin{abstract}
This paper considers the challenging task of realtime extended object tracking using cluttered measurements from laser range scanners. The performance of the recently proposed Box Particle Filter (Box PF) algorithm is evaluated utilising real measurements from laser range scanners obtained within a prototype security system replicating an airport corridor.

The problem is expressed as the joint estimation of both state and parameters of an extended target. Circularly and elliptically shaped targets are considered. Promising results are presented.
\end{abstract}

\section{INTRODUCTION}

The problem of tracking an extended object has been extensively studied in the recent years. Such objects of interest could be individual targets or a formation (considered as one target) such as a convoy of vehicles, a formation of aircrafts, a fleet of ships, a crowd of people, etc. The particularity here is that such objects are giving rise to many measurements and need sophisticated methods to cope with them. The proximity of the object and the resolution of the sensors may often be sufficient for the observer to infer some valuable information from the measurements about the shape and the size of the target's extent.

Usually the problem is formulated as a joint estimation of kinematic states and parameters, where the parameters relate to the extent of the object of interest [1], [2], [3], [4], [5], [6], [7] and the main methodology is the Bayesian framework. Various filters have been developed for extended target tracking: particle track-before-detect filters [8], cluster based approaches [9], [10], Poisson spatial models combined with particle filters (PFs) [11], [12], [13], [14] and mixture Kalman filters combined with data augmentation [1].

Recently various Probabilistic Hypothesis Density (PHD) filters for extended object tracking have been proposed, i.e. [15]. As an alternative, various interval algorithms have been developed, mainly for linear systems and linear measurements [16], [17], [18]. This paper proposes a Box Particle Filter (Box PF) framework for extended object tracking. The Box Particle filter is developed in [19] and applied for localisation problems. The theoretical justification of the Box PF approach is derived in [20], [21]. The Box PF has also been applied to filtering problems within the random finite set statistics approach in [22] and in particular as a more efficient implementation of a Bernouli PF.

In the present paper the Box PF addresses the problem of extended target tracking. The main contribution of this work is in presenting a general form for calculating the likelihood function based on solving a Constraint Satisfaction Problem (CSP) and the performance evaluation of the algorithm using real data from laser range scanners.

The rest of this paper is organised as follows. Section II gives the main idea behind the Box PF. Section III presents the formulation of the problem within the Bayesian framework. Section IV introduces the necessary theoretical background from interval analysis. The Box Particle Filtering algorithm for extended target tracking is presented in Section V. The evaluation scenarios are described in Section VI, the results are given in Section VII and the conclusions in Section VIII.

\section{Main IdeA OF THE Box PARTiCle Filtering}

The main idea of the Box PF is to replace the point particles with region-particles, also called boxes. This approach is suitable for dealing with various types of uncertainties in the measurements: i.e. interval, stochastic and data association uncertainties [22]. These three types of uncertainties can be dealt with the same prediction and correction steps as in the case with the generic PF. However, there are some significant differences.

The prediction step is performed in a similar way as in the classical PF, however, it is with respect to box particles. When a box particle is propagated via a non-linear function, the image of it is not necessarily a box particle. Hence, a function, called inclusion function, introduced in the previous section, is applied to convert the predicted region into a box particle. The measurement update step requires the calculation of the generalised likelihood function. Since the measurement noise is supposed to be bounded, a likelihood box is defined as a set containing the measurement and the noise boundaries. This generalised measurement likelihood function is calculated in a different way compared to the classical PF [23]. The measurement update step is based on finding the minimum boxes inside the box particles, consistent with the likelihood box. This is done with a procedure called contraction which 
removes inconsistent parts between the box particles and the likelihood box. When there is no consistency between the box state particle and the likelihood box, the likelihood value is set to zero. This case commonly happens when a clutter measurement is contracted with the box state particle. There are different methods for performing the contraction step [24]. The resampling step is used to introduce variety. However, the resampling step in the box PF differs from the resampling step of the generic PF. The resampling step in the Box PF can be performed by a division of box particles [22] or by other techniques.

\section{PROBLEM FoRMULATION WITHIN THE BAYESIAN FRAMEWORK}

The system dynamics and the sensor equations have the following general form:

$$
\begin{aligned}
\boldsymbol{x}_{k} & =f\left(\boldsymbol{x}_{k-1}, \boldsymbol{\eta}_{k}\right), \\
\boldsymbol{z}_{k} & =h\left(\boldsymbol{x}_{k}, \boldsymbol{w}_{k}\right),
\end{aligned}
$$

where $\boldsymbol{x}_{k}=\left(\boldsymbol{X}_{k}^{T}, \boldsymbol{\Theta}_{k}^{T}\right)^{T} \subset \mathbb{R}^{n_{x}}$ and $\boldsymbol{z}_{k} \subset \mathbb{R}^{n_{z}}$ are the unknown system state vector and the measurement vector, respectively, at time step $k, k=1,2, \ldots, K$. The maximum number of time steps is given by $K ; n_{x}$ is the dimension of $\boldsymbol{x}_{k}$ and $n_{z}$ is the dimension of $z_{k}$. The notation $(\cdot)^{T}$ is used for the transpose operator. The system function $f(\cdot)$ and measurement function $h(\cdot)$ are nonlinear in general. The vector $\boldsymbol{x}_{k}$ consists of the object kinematic state vector $\boldsymbol{X}_{k} \subset \mathbb{R}^{n_{X}}$ and object extent, characterised by the parameters vector $\boldsymbol{\Theta}_{k} \subset \mathbb{R}^{n_{\Theta}}$, where $n_{\Theta}$ is the number of parameters to be estimated. The system (kinematic state and parameters) noise and measurement noise are given, respectively, by: $\boldsymbol{\eta}_{k}=\left(\boldsymbol{\eta}_{X, k}^{T}, \boldsymbol{\eta}_{\Theta, k}^{T}\right)^{T}$ and $\boldsymbol{w}_{k}$.

According to the Bayesian framework the state vector is obtained in a recursive way based on the following equations, for the prediction

$$
p\left(\boldsymbol{x}_{k} \mid \boldsymbol{z}_{1: k-1}\right)=\int_{\mathbb{R}^{n_{x}}} p\left(\boldsymbol{x}_{k} \mid \boldsymbol{x}_{k-1}\right) p\left(\boldsymbol{x}_{k-1} \mid \boldsymbol{z}_{1: k-1}\right) d \boldsymbol{x}_{k-1}
$$

and respectively for the update

$$
p\left(\boldsymbol{x}_{k} \mid \boldsymbol{z}_{1: k}\right)=\frac{p\left(\boldsymbol{z}_{k} \mid \boldsymbol{x}_{k}\right) p\left(\boldsymbol{x}_{k} \mid \boldsymbol{z}_{1: k-1}\right)}{p\left(\boldsymbol{z}_{k} \mid \boldsymbol{z}_{1: k-1}\right)},
$$

where $p\left(\boldsymbol{x}_{k} \mid \boldsymbol{z}_{1: k-1}\right)$ is the prior state probability density function (pdf), $p\left(\boldsymbol{x}_{k} \mid \boldsymbol{x}_{k-1}\right)$ is the state transition pdf, $p\left(\boldsymbol{x}_{k-1} \mid \boldsymbol{z}_{1: k-1}\right)$ is the posterior state pdf at time step $k-1$, $p\left(\boldsymbol{x}_{k} \mid \boldsymbol{z}_{1: k}\right)$ is the posterior state pdf at time step $k, p\left(\boldsymbol{z}_{k} \mid \boldsymbol{x}_{k}\right)$ is the likelihood function and $p\left(\boldsymbol{z}_{k} \mid \boldsymbol{z}_{1: k-1}\right)$ is a normalisation factor.

\section{BACKGROUND FROM INTERVAL ANALYSIS}

A real interval, $[x]=[\underline{x}, \bar{x}]$ is defined as a closed and connected subset of the set $\mathbb{R}$ of real numbers. The lower bar is the minimum value of a quantity and the upper bar is the maximum value of a quantity. In a vector form, a box $[\boldsymbol{x}]$ of $\mathbb{R}^{n_{x}}$ is defined as a Cartesian product of $n_{x}$ intervals: $[\boldsymbol{x}]=\left[x_{1}\right] \times\left[x_{2}\right] \cdots \times\left[x_{n}\right]=\times_{i=1}^{n_{x}}\left[x_{i}\right]$. In this paper, the operator $|[]$.$| denotes the size |[\boldsymbol{x}]|$ of a box $[\boldsymbol{x}]$. The underlying concept of interval analysis is to deal with intervals of real numbers instead of dealing with real numbers. For that purpose, elementary arithmetic operations, e.g., $+,-, *, \div$, etc., as well as operations between sets of $\mathbb{R}^{n}$, such as $\subset, \supset, \cap, \cup$, etc., have been naturally extended to interval analysis context.

In addition, a lot of research has been performed with the so called inclusion functions [24]. An inclusion function $[f]$ of a given function $f$ is defined such that the image of a box $[\boldsymbol{x}]$ is a box $[f]([\boldsymbol{x}])$ containing $f([\boldsymbol{x}])$. The goal of inclusion functions is to work only with intervals, to optimise the interval enclosing the real image set and then to decrease the pessimism when intervals are propagated.

Often constraints have to be fulfilled which requires to solve the CSPs. A CSP often denoted $\mathcal{H}$ can be written as:

$$
\mathcal{H}:(\boldsymbol{f}(\boldsymbol{x})=\mathbf{0}, \boldsymbol{x} \in[\boldsymbol{x}]) .
$$

Equation (5) can be interpreted as follows: find the optimal box enclosure of the set of vector $\boldsymbol{x}$ belonging to a given prior domain $[\boldsymbol{x}] \subset \mathbb{R}^{n}$ satisfying a set of $m$ constraints $\boldsymbol{f}$ (with $\boldsymbol{f}$ a multivalued function, i.e., $\mathbf{f}=\left(f_{1}, f_{2}, \cdots, f_{m}\right)^{T}$, where the $f_{i}$ are real valued functions). The solution set of $\mathcal{H}$ is defined as:

$$
\mathcal{S}=\{\boldsymbol{x} \in[\boldsymbol{x}] \mid \boldsymbol{f}(\boldsymbol{x})=\mathbf{0}\} .
$$

The contraction $\mathcal{H}$ means replacing $[\boldsymbol{x}]$ by a smaller domain $[\boldsymbol{x}]^{\prime}$ such that $\mathcal{S} \subseteq[\boldsymbol{x}]^{\prime} \subseteq[\boldsymbol{x}]$. A contractor for $\mathcal{H}$ is any operator that can be used to contract $\mathcal{H}$. Several methods for building contractors are described in [24, Chapter 4], e.g. Gauss elimination, the Gauss-Seidel algorithm, linear programming. Each of these methods may be more suitable to some types of CSP. Although the approaches presented in this work are not limited to any particular contractor, a general and well known contraction method, the Constraints Propagation (CP) technique is used in this paper. The main advantages of the CP method is its efficiency in the presence of high redundancy of data and equations. The $\mathrm{CP}$ method is also known to be simple and, most importantly, to be independent of nonlinearities.

\section{Box Particle Filtering Algorithm for EXTENDED TARGET TRACKING}

The Box PF algorithm for extended target tracking consists of the following steps:

0. Initialise the box particles $\left[\boldsymbol{x}_{0}^{(p)}\right]=\hat{\boldsymbol{x}}_{0}+\left[-3 \boldsymbol{\sigma}_{0},+3 \boldsymbol{\sigma}_{0}\right]$, $p=1, \ldots, N$. The available information about the target state is contained within the state vector $\left[\hat{\boldsymbol{x}}_{0}\right]=\left(\left[\boldsymbol{X}_{0}\right]^{T},\left[\boldsymbol{\Theta}_{0}\right]^{T}\right)^{T} \subset$ $\mathbb{R}^{n_{x}}$ and the standard deviation parameter vector $\boldsymbol{\sigma}_{0} \subset \mathbb{R}^{n_{x}}$.

Repeat for $K$ time steps, $k=1, \ldots K$, the following steps:

\section{Prediction}

1. Propagate the box particles $\left[\boldsymbol{x}_{k-1}^{(p)}\right]$ through the state evolution model $\left[\boldsymbol{x}_{k \mid k-1}^{(p)}\right]=[f]\left(\left[\boldsymbol{x}_{k-1}^{(p)}\right],\left[\boldsymbol{\eta}_{k}\right]\right)$ to obtain the predicted box particles $\left[\boldsymbol{x}_{k \mid k-1}^{(p)}\right]$. An inclusion function ensures the resulting state vector is again a box.

\section{Update}


2. Upon receiving the measurements from the sensor(s) reduce their number to those $M$ that are within the observability region of the sensor(s).

3. To take into account the uncertainty of the sensor(s), form intervals around the measurements $\boldsymbol{z}_{k}^{j}$, thus obtaining the measurement likelihood boxes $\left[\boldsymbol{z}_{k}^{j}\right]$, i.e. $\left[\boldsymbol{z}_{k}^{j}\right]=\boldsymbol{z}_{k}^{j}+[-\Delta,+\Delta]$. If the standard deviation of the measurement error $\sigma_{z}$ is available the added interval of uncertainty can be $\left[-3 \sigma_{z},+3 \sigma_{z}\right]$ for example. Often, the case is that an error interval $\pm \Delta$ is available in the technical sheet of the measurement device instead. Using this interval is a convenient way of obtaining the likelihood boxes.

4. Transform the measurements to a coordinate system consistent with the coordinate system of the boxes, i.e. from polar with respect to the sensor to Cartesian with respect to the center of the coordinate system. Apply an inclusion function after the transformation.

5. Contract the measurements with the box particles. Only the contracted measurements are used in the next steps. They are refereed as contracted measurement boxes. As a result, $M_{c}^{(p)} \leq M$ contracted measurements boxes remain associated with the box particle $p$.

6. Calculate the likelihood terms $p\left(\left[\boldsymbol{z}_{k}^{j}\right] \mid\left[\boldsymbol{x}_{k}^{(p)}\right]\right), \forall$ box particles $p=1, \ldots, N$ and $\forall$ contracted measurement boxes $j=1, \ldots M_{c}^{(p)}$, as the area included within the contracted measurement boxes divided by the area covered by their associated box particles.

7. For each box particle $p$ perform contraction based on each of the contracted measurement boxes $m=1, \ldots, M_{c}^{(p)}$. Here we perform it using the CSP technique. This results in obtaining $M_{c}^{(p)}$ contracted box particles $\left\{\left[\boldsymbol{x}_{k}^{\left((p)_{m}\right)}\right]\right\}_{m=1}^{M_{c}^{(p)}}$ from each of the $p$ box particles.

8. Obtain an estimate for each of the contracted box particles $\left[\boldsymbol{x}_{k, c}^{(p)}\right], p=1, \ldots, N$ as a weighted sum of the contracted box particles $p_{m}, m=1, \ldots, M_{c}^{(p)}$ using the weights obtained in step 5.

9. Calculate the weights for each of the contracted box particles:

9.1. Calculate the terms $p\left(\left[\boldsymbol{z}_{k}^{j}\right] \mid\left[\boldsymbol{x}_{k, c}^{(p)}\right]\right), \forall$ contracted box particles $p=1, \ldots, N$ and $\forall$ contracted measurements $j=$ $1, \ldots M_{c}^{(p)}$, as the area included within the contracted measurement boxes divided by the area covered by their associated contracted box particles.

9.2. Calculate the weights $w_{k}^{(p)}, p=1, \ldots, N$ using terms from step 8.1. and the spatial distribution model derived in [12], under the assumption that the number of measurements originating from the target and the number of clutter points have Poisson distribution:

$$
\begin{gathered}
p\left(\left[\boldsymbol{z}_{k}\right] \mid\left[\boldsymbol{x}_{k}^{(p)}\right]\right)=\prod_{j=1}^{M}\left(1+\frac{\lambda_{T}}{\rho} p\left(\left[\boldsymbol{z}_{k}^{j}\right] \mid\left[\boldsymbol{x}_{k, c}^{(p)}\right]\right)\right), \\
w_{k}^{(p)}=w_{k-1}^{(p)} \frac{p\left(\left[\boldsymbol{z}_{k}\right] \mid\left[\boldsymbol{x}_{k}^{(p)}\right]\right)}{\sum_{p=1}^{N} p\left(\left[\boldsymbol{z}_{k}\right] \mid\left[\boldsymbol{x}_{k}^{(p)}\right]\right)},
\end{gathered}
$$

where $\lambda_{T}$ is the average number of the measurements originating from the target and $\rho$ is the clutter density. The same Poisson assumption is adopted in this work.

10. Obtain a box estimate for the state of the extended target as a weighted sum of all particles:

$$
\left[\hat{\boldsymbol{x}}_{k}\right]=\sum_{p=1}^{N} w_{k}^{(p)}\left[\boldsymbol{x}_{k}^{(p)}\right]
$$

and a (non-interval) estimate $\hat{\boldsymbol{x}}_{k}$ for the extended shape using the mid-points of the box estimated of the state vector $\left[\hat{\boldsymbol{x}}_{k}\right]$.

\section{Resampling}

11. Computing the effective sample size $N_{\text {eff }}=$ $1 / \sum_{p=1}^{N}\left(w_{k}^{(p)}\right)^{2}$. Choose a threshold, for example $N_{\text {th }}=2 N / 3$.

12. If $N_{\text {eff }}<N_{\text {th }}$ perform resampling using a modified version of the Sequential Importance Resampling (SIR) algorithm, in which the uncertainty regions of the selected box particles are reduced to randomly selected portions of the initial uncertainty regions. The weights are set to $w_{k}^{(p)}=1 / N$.

\section{EVALUATION SCENARIOS}

The performance of the Box PF algorithm for extended object tracking is evaluated using data obtained within a prototype security system replicating an airport corridor. This data consists of range and bearing components obtained by three laser rangefinder devices. The measurement devices are positioned at three key locations, marked with crossed squares, in a curved corridor (see Fig. 1). Two different scenarios are considered. The first scenario is with a single person holding a cylindrical object with radius equal to $18 \mathrm{~cm}$ around his body at the height of the sensors, referred as circular object scenario from here on. In this scenario the target is visible from at least one sensor (usually from two). The second scenario is originally a multiple target tracking scenario with several people which in order to relate to our problem we use partially from the point of initial entry of the first person until the first crossing point of that person with another one walking in the scene. This scenario will be referred as the human body scenario. In this case the people are moving in and out of the area visible by the sensors. Because of intercrossing the targets on the scene experience obscurations or occlusions at different sections of their trajectories. The sensors are positioned around the height of the hip, i.e. the measurements originate from the body and from the moving limbs of the people. In that case the walking people could be approximated with circles with radius approximately $15-20 \mathrm{~cm}$. No exact ground truth is available in both cases. Nevertheless, the information from video cameras positioned in the corridor gives a sort of ground truth for the trajectory of movement of the targets.

\section{A. Model of the Extended Targets}

We look at a two-dimensional case, where the state vector consists of position coordinates and velocity of the centre of 
the extent. Then the kinematic state vector is in the from

$$
\begin{aligned}
{\left[\boldsymbol{X}_{k}\right] } & =\left(\left[x_{k}\right],\left[\dot{x}_{k}\right],\left[y_{k}\right],\left[\dot{y}_{k}\right]\right)^{T} \\
& =\left(\left[\underline{x_{k}}, \overline{x_{k}}\right],\left[\underline{\dot{x}_{k}}, \overline{\dot{x}_{k}}\right],\left[\underline{y_{k}}, \overline{y_{k}}\right],\left[\underline{\dot{y}_{k}}, \overline{\dot{y}_{k}}\right]\right)^{T} .
\end{aligned}
$$

The interval parameter vector, in its general form, is

$$
\begin{aligned}
{\left[\boldsymbol{\Theta}_{k}\right] } & =\left(\left[\theta_{1, k}\right],\left[\theta_{2, k}\right], \cdots,\left[\theta_{n_{\Theta}, k}\right]\right)^{T} \\
& =\left(\left[\underline{\theta_{1, k}}, \overline{\theta_{1, k}}\right],\left[\underline{\theta_{2, k}}, \overline{\theta_{2, k}}\right], \cdots,\left[\underline{\theta_{n_{\Theta}, k}}, \overline{\theta_{n_{\Theta}, k}}\right]\right)^{T} .
\end{aligned}
$$

Here we consider a circularly-shape approximation of the extended target, therefore the parameter vector holds only the radius of that circle

$$
\left[\boldsymbol{\Theta}_{k}\right]=\left(\left[r_{k}\right]\right)=\left(\left[\underline{r_{k}}, \overline{r_{k}}\right]\right) .
$$

The motion of the interval centre of the extended target is modelled by the nearly constant velocity model [25], [26]. The evolution model for the interval state of the target is

$$
\left[\boldsymbol{X}_{k}\right]=\boldsymbol{A}\left[\boldsymbol{X}_{k-1}\right]+\boldsymbol{\Gamma}\left[\boldsymbol{\eta}_{X, k}\right] .
$$

The state transition matrix $\boldsymbol{A}_{1}=\left(\begin{array}{cc}1 & T_{s} \\ 0 & 1\end{array}\right)$ for the two dimensional case is given by $\boldsymbol{A}=\operatorname{diag}\left(\boldsymbol{A}_{1}, \boldsymbol{A}_{1}\right), \boldsymbol{\Gamma}=$ $\left(\begin{array}{cccc}T_{s}^{2} / 2 & T_{s} & 0 & 0 \\ 0 & 0 & T_{s}^{2} / 2 & T_{s}\end{array}\right)^{T}$ and $T_{s}$ is the sampling interval. The system dynamics noise $\left[\boldsymbol{\eta}_{X, k}\right]$ is characterised by the standard deviation parameters $\sigma_{x}$ and $\sigma_{y}$. Then the system dynamics noise is represented as a Gaussian noise process with covariance $\boldsymbol{Q}=\operatorname{diag}\left(\boldsymbol{Q}_{1} \sigma_{x}^{2}, \boldsymbol{Q}_{1} \sigma_{y}^{2}\right)$, where $\boldsymbol{Q}_{1}=$ $\left(\begin{array}{cc}T_{s}^{4} / 4 & T_{s}^{3} / 2 \\ T_{s}^{3} / 2 & T_{s}^{2}\end{array}\right)$. The evolution for the extent is assumed to be a random walk model, described by the equation

$$
\left[\boldsymbol{\Theta}_{k}\right]=\left[\boldsymbol{\Theta}_{k-1}\right]+\left[\boldsymbol{\eta}_{\Theta, k}\right],
$$

where the interval parameters noises $\left[\boldsymbol{\eta}_{\Theta, k}\right]$ are characterised by $\sigma_{\Theta} \in \mathbb{R}^{n_{\Theta}}$. Then again, the augmented interval state vector is $\left[\boldsymbol{x}_{k}\right]=\left(\left[\boldsymbol{X}_{k}\right]^{T},\left[\boldsymbol{\Theta}_{k}\right]^{T}\right)^{T}$.

\section{B. Observation Model}

The collected measurements consist of range and bearing. The number of measurements $M$ obtained at each time step from an active sensor consists of $M_{T}$ measurements originating from the target and $M_{C}$ clutter measurements, i.e. $M=M_{T}+M_{C}$. The measurement equation for the measurement point $j, j=1, \ldots, M$ is of the form:

$$
\boldsymbol{z}_{k}^{j}=h\left(\boldsymbol{x}_{k}, \boldsymbol{w}_{k}^{j}\right) \text {. }
$$

The range and bearing components are described respectively:

$$
\begin{gathered}
d_{k}^{j}=\sqrt{x_{k}^{j 2}+y_{k}^{j 2}}+w_{d, k}^{j}, \\
\beta_{k}^{j}=\tan ^{-1} \frac{y_{k}^{j}}{x_{k}^{j}}+w_{\beta, k}^{j},
\end{gathered}
$$

where $x_{k}^{j}$ and $y_{k}^{j}$ denote the Cartesian coordinates of the actual point of the source which generates the measurement in the case of two dimensional space. The measurement noise

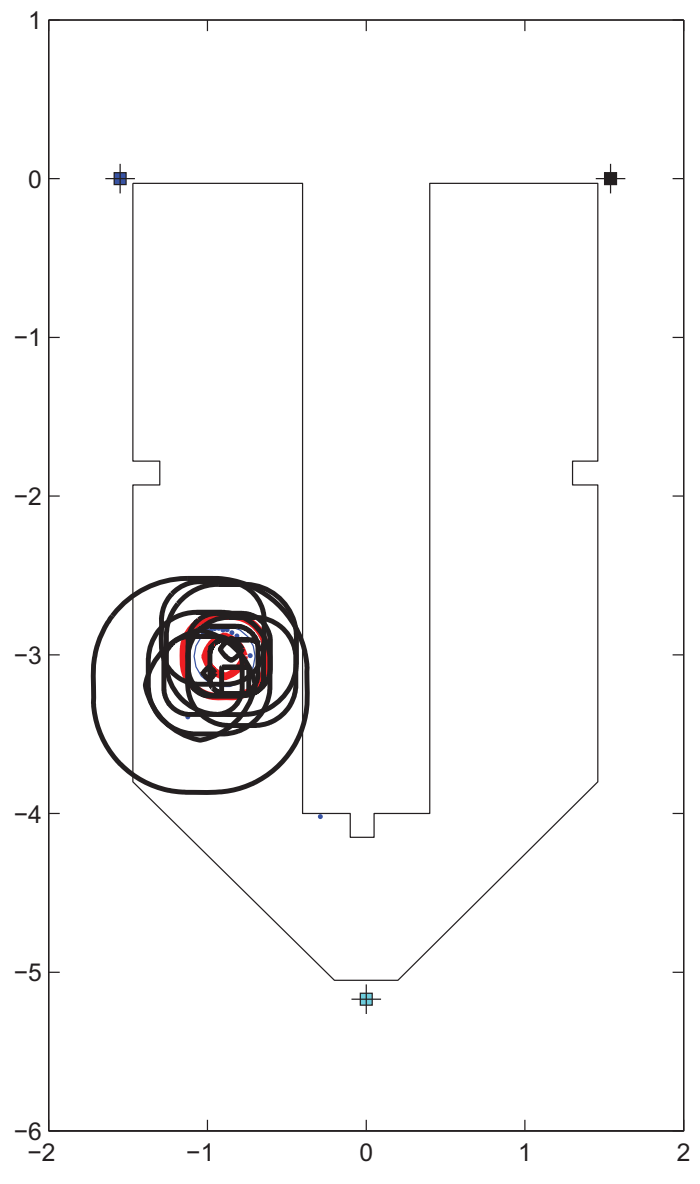

Fig. 1. Testing scenario. The three laser scanner devices are indicated with crossed boxes.

$\boldsymbol{w}_{k}^{j}=\left(w_{d, k}^{j}, w_{\beta, k}^{j}\right)^{T}$, is assumed (but not restricted) to be Gaussian, with a known covariance matrix $\boldsymbol{R}=\operatorname{diag}\left(\sigma_{d}^{2}, \sigma_{\beta}^{2}\right)$.

The interval measurements vector is $\left[\boldsymbol{z}_{k}^{j}\right]=\left(\left[d_{k}^{j}\right],\left[\beta_{k}^{j}\right]\right)^{T}$, where $\left[d_{k}^{j}\right]$ is the interval range and $\left[\beta_{k}^{j}\right]$ is the interval bearing of the measurement point $j$. One way to describe these components is:

$$
\begin{aligned}
& {\left[d_{k}^{j}\right]=d_{k}^{j}+\left[-3 \sigma_{d},+3 \sigma_{d}\right],} \\
& {\left[\beta_{k}^{j}\right]=\beta_{k}^{j}+\left[-3 \sigma_{\beta},+3 \sigma_{\beta}\right] .}
\end{aligned}
$$

\section{ViI. Performance Evaluation}

The performance of the algorithm is evaluated by averaging over 500 repetitive runs for the two scenarios described above. The estimated trajectories for the circular target scenario and for the human body scenario are presented in Fig. 3(a) and Fig. 3(b), respectively. Fig. 3(c) and Fig. 3(d) present the averaging for the estimated radius. Fig. 3(a) to Fig. 3(d) give both interval and non-interval estimation graphs, where the non-interval results are obtained as the mean of the intervals. The performance is further evaluated based on the volume of the interval shape and the inclusion of the measurements within that interval shape show in Fig. 4 and Fig. 5. 


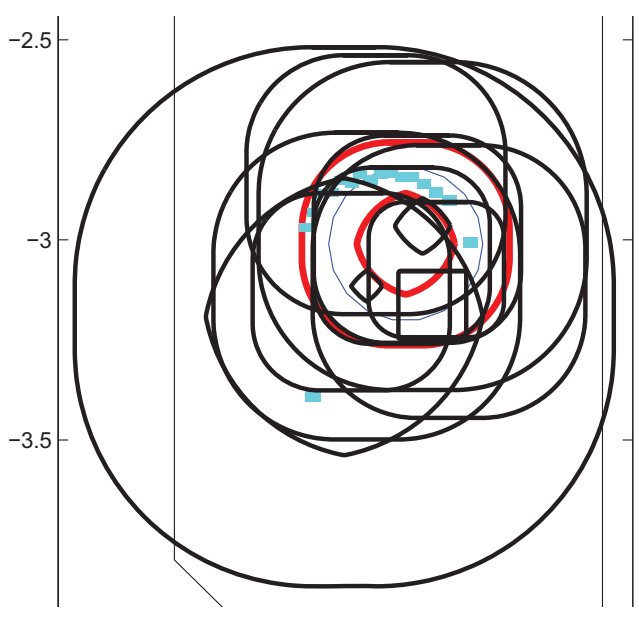

Fig. 2. The box particles are given with black lines, the estimated interval shape is in red colour the measurement likelihood boxes are in cyan and the non-interval estimate is given in blue

During the first $\sim 10$ time steps the circular target is entering the scene. Fig. 3(a) shows that this leads to decreasing the uncertainty within the target's state vector after the initial blow up of the box particles. This is related to the volume of the interval shape, which decreases, as seen in Fig. 4. As seen in Fig. 3(c) the estimate of the target's radius is within the expected range - an interval of $\sim[7 \mathrm{~cm}, 27 \mathrm{~cm}]$, and mean of $\sim 17 \mathrm{~cm}$. Due to the nature of the sensors, the number of the measurements inside the interval shape is higher when the object is closer to any of the sensors. Nevertheless, the percentage of measurements that are inside the region of the interval shape remains higher than $90 \%$ throughout the tracking process in this scenario, see Fig. 4.

Similar behaviour is observed in the human body scenario. An initial decrease of the interval uncertainty to a certain interval size is observed, once the target enters the scene. The difference here is the presence of up to 4 targets in the scene at the same time. It leads to lower percentage of measurements ( $\sim 1 / 4$ of the initial $90 \%)$ within the estimated interval target shape after three other targets enter the scene, see Fig. 5. That happens between time steps 30 to 50 . The estimated radius is shown in Fig. 3(d) to be at an interval of $\sim[8 \mathrm{~cm}, 25 \mathrm{~cm}]$, with a mean of $\sim 16.5 \mathrm{~cm}$.

\section{CONCLUSIONS}

This paper presents a Box Particle Filter for extended target tracking in the presence of clutter and validates its performance based on real data. Two different scenarios are considered and the results show precise and effective estimation of the extended target state. Both an interval and a noninterval (punctual) estimation of the target state is obtained as an output of the filter. The Box Particle Filter appears to be a promising technique for extended target tracking an will be a subject to further studies.

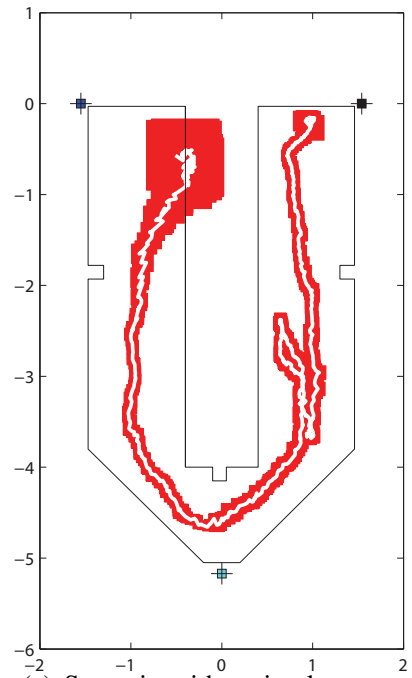

(a) Scenario with a circular target

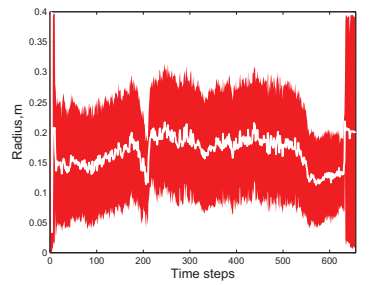

(c) Estimated radius, circular target
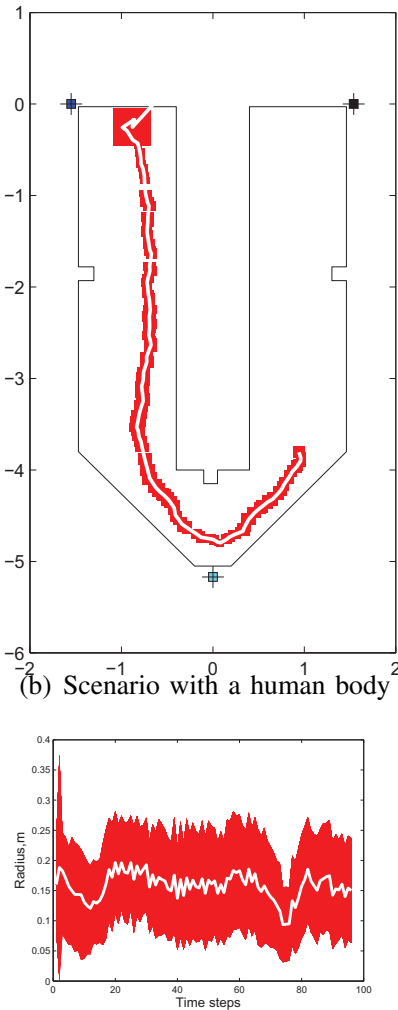

(d) Estimated radius, human body
Fig. 3. Performance evaluation for circular target scenario (a), (b) and for human body scenario (c), (d).
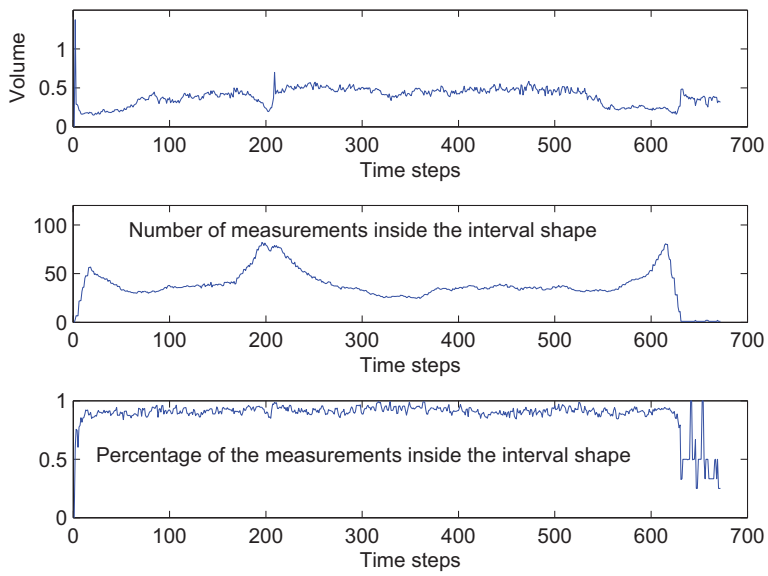

Fig. 4. Performance measures, circular target

\section{ACKNOWLEDGMENTS}

We acknowledge the support from the [European Community] Seventh Framework Programme [FP7/2007- 2013] under grant agreement No 238710 (Monte Carlo based Innovative Management and Processing for an Unrivalled Leap in Sensor Exploitation). 

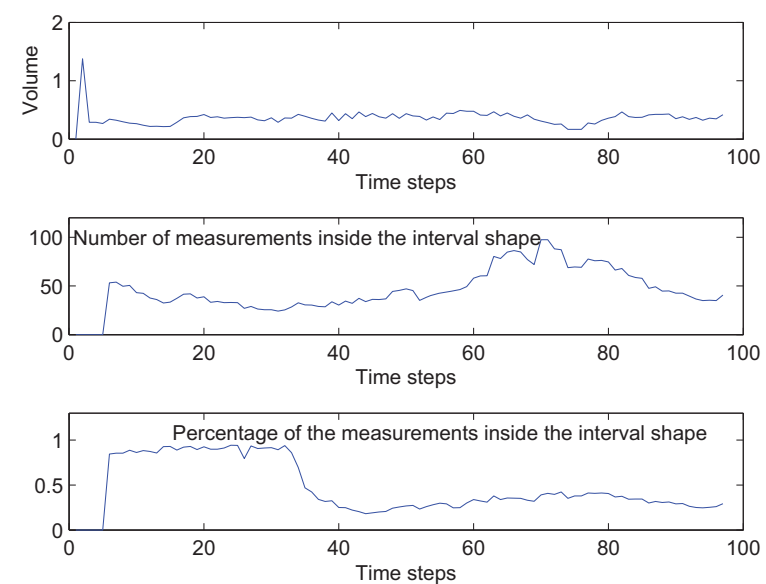

Fig. 5. Performance measures, human body scenario

\section{REFERENCES}

[1] D. Angelova and L. Mihaylova, "Extended object tracking using Monte Carlo methods," IEEE Transactions on Signal Processing, vol. 56, no. 2, pp. 825-832, 2008

[2] — "Extended object tracking using mixture Kalman filtering," in Numerical Methods and Applications. Lecture Notes in Computer Science. T. Boyanov et al. (Eds.), May 2007, pp. 122-130. [Online]. Available: http://www.springerlink.com/content/lj3074n372807754

[3] M. Feldmann, D. Fränken, and W. Koch, "Tracking of extended objects and group targets using random matrices," IEEE Transactions on Signal Processing, vol. 59, no. 4, pp. 1409 -1420, april 2011.

[4] M. Baum, M. Feldmann, D. Fränken, U. D. Hanebeck, and W. Koch, "Extended object and group tracking: A comparison of random matrices and random hypersurface models," in GI Jahrestagung (2), 2010, pp. 904-906.

[5] W. Koch and M. Feldmann, "Cluster tracking under kinematical constraints using random matrices," Robotics and Autonomous Systems, vol. 57, no. 3, pp. 296 - 309, 2009.

[6] M. Feldmann and D. Fränken, "Tracking of extended objects and group targets using random matrices - a new approach," in Proc. of the 2008 11th International Conf. on Information Fusion,, 2008, pp. $1-8$.

[7] W. Koch and R. Saul, "A Bayesian approach to extended object tracking and tracking of loosely structured target groups," in Proc. of the 8th International Conf. on Inform. Fusion. ISIF, 2005.

[8] Y. Boers, H. Driesen, J. Torstensson, M. Trieb, R. Karlsson, and F. Gustafsson, "Track-before-detect algorithm for tracking extended targets," IEE Proceedings on Radar, Sonar and Navigation, vol. 153, no. 4 , pp. $345-351,2006$.

[9] O. Drummond, S. Blackman, and G. Petrisor, "Tracking clusters and extended objects with multiple sensors," in Proc. of SPIE, Vol. 1305, Signal and Data Processing of Small Targets 1990, 1990, pp. 362-375.

[10] A. Swain and D. Clark, "Extended object filtering using spatial independent cluster processes," in Proceedings of the 13th Conference on Information Fusion (FUSION), july 2010, pp. 1 -8.

[11] D. Salmond and N. Gordon, "Group and extended object tracking," in Proc. IEE Colloquium on Target Tracking: Algorithms and Applications, 1999, pp. $16 / 1$ - 16/4.

[12] K. Gilholm and D. Salmond, "Spatial distribution model for tracking extended objects," IEE Proc.-Radar, Sonar Navig., vol. 152, no. 5, pp. 364-371, 2005.

[13] K. Gilholm, S. Godsill, S. Maskell, and D. Salmond, "Poisson models for extended target and group tracking," in Proceedings of SPIE 5913, 2005 .

[14] J. Vermaak, N. Ikoma, and S. Godsill, "Sequential Monte Carlo framework for extended object tracking," IEE Proc.-Radar, Sonar Navig., vol. 152, no. 5, pp. 353-363, 2005.
[15] K. Granström, C. Lundquist, and U. Orguner, "Tracking rectangular and elliptical extended targets using laser measurements," in Proceedings of the International Conference on Information Fusion, Chicago, IL, USA, Jul. 2011, pp. $592-599$.

[16] M. Baum and U. Hanebeck, "Shape tracking of extended objects and group targets with star-convex RHMs," in Proceedings of the International Conference on Information Fusion, 2011.

[17] M. Baum and U. D. Hanebeck, "Extended object tracking based on combined set-theoretic and stochastic fusion," in Proc. of the International Conf. on Information Fusion, 2009.

[18] M. Baum and U. Hanebeck, "Random hypersurface models for extended object tracking," in Proc. of the IEEE International Symp. on Signal Processing and Information Technology (ISSPIT), 2009, pp. $178-183$.

[19] F. Abdallah, A. Gning, and P. Bonnifait, "Box particle filtering for nonlinear state estimation using interval analysis," Automatica, vol. 44, no. 3, pp. 807-815, 2008.

[20] A. Gning, L. Mihaylova, and F. Abdallah, "Mixture of uniform probability density functions for nonlinear state estimation using interval analysis," in Proc. of 13th International Conference on Information Fusion. ISIF, Edinburgh, UK, 2010.

[21] A. Gning, L. Mihaylova, F. Abdallah, and B. Ristic, "Particle filtering combined with interval methods for tracking applications," in Integrated Tracking, Classification, and Sensor Management: Theory and Applications, M. Mallick, V. Krishnamurthy, and B.-N. Vo, Eds. John Wiley \& Sons, 2012.

[22] A. Gning, B. Ristic, and L. Mihaylova, "Bernouli/ box-particle filters for detection and tracking in the presence of triple measurement uncertainty," IEEE Transactions on Signal Processing, vol. 60, no. 5, pp. 2138 - 2151, 2012.

[23] B. Ristic, "Bayesian estimation with imprecise likelihoods: Random set approach," Signal Processing Letters, IEEE, vol. 18, no. 7, pp. 395 -398, july 2011

[24] L. Jaulin, M. Kieffer, O. Didrit, and E. Walter, Applied Interval Analysis. Springer-Verlag, 2001.

[25] X. R. Li and V. Jilkov, "A survey of maneuveuvering target tracking. Part I: Dynamic models," IEEE Trans. on Aerosp. and Electr. Systems, vol. 39, no. 4, pp. 1333-1364, 2003.

[26] Y. Bar-Shalom and X. Li, Estimation and Tracking: Principles, Techniques and Software. Artech House, 1993. 